\title{
Multi-continued fraction algorithm on multi-formal Laurent series
}

by

\section{Zongduo Dai, Kunpeng Wang and Dingfeng Ye (Beijing)}

1. Introduction. Continued fractions $[8,14]$ are a useful tool in many number theoretical problems and in numerical computing. It is well known that the simple continued fraction expansion of a single real number gives the best solution to its rational approximation problem. Many people have contrived to construct multidimensional continued fractions in dealing with the rational approximation problem for multi-reals. One construction is the Jacobi-Perron algorithm (JPA) (see [1]). This algorithm and its modifications have been extensively studied $[6,7,10,13]$. These algorithms are adapted to study the same problem for multi-formal Laurent series $[2,4,11,12]$. But none of them guarantees the best rational approximation in general. In this paper, we deal with the multi-rational approximation problem over the formal Laurent series field $F\left(\left(z^{-1}\right)\right)$ : given an element $\underline{r} \in F\left(\left(z^{-1}\right)\right)^{m}$, find $\underline{p} \in F[z]^{m}$ and $q \in F[z]$ such that $\underline{p} / q$ approximates $\underline{r}$ as close as possible while $\operatorname{deg}(q)$ is bounded.

We propose a new continued fraction algorithm for multi-formal Laurent series. It is proved that this algorithm guarantees best rational approximations for multi-formal Laurent series.

The paper is organized as follows: Section 2 deals with the indexed valuation of $F\left(\left(z^{-1}\right)\right)^{m}$. Section 3 contains the detailed definition of the problem of optimal rational approximation of multi-formal Laurent series. Section 4 proposes an algorithm called multidimensional continued fraction algorithm (m-CFA, for short), which produces a multi-continued fraction expansion $C(\underline{r})$ for any given multi-formal Laurent series $\underline{r}$. Section 5 shows that $C(\underline{r})$ satisfies three basic conditions. Section 6 states the main results of this pa-

2000 Mathematics Subject Classification: 11A55, 11B37, 11J70, 11T71.

Key words and phrases: multidimensional formal Laurent series, multidimensional continued fraction, rational approximation, valuation.

This work is partly supported by NSFC (Grant No. 60473025), and the National 973 Project (Grant No. 1999035804). 
per: $C(\underline{r})$ provides optimal rational approximations to $\underline{r}$. In Section 7 we complete all proofs.

2. Indexed valuation over $F\left(\left(z^{-1}\right)\right)$. Denote by $\mathbb{Z}$ the ring of integers, by $F$ an arbitrary field, by $m$ a positive integer, and by $Z_{m}$ the set $\{1, \ldots, m\}$. Let $F[z]$ be the polynomial ring over $F, F(z)$ the rational fraction field over $F$, and

$$
F\left(\left(z^{-1}\right)\right)=\left\{\sum_{i \geq t} a_{i} z^{-i} \mid t \in \mathbb{Z}, a_{i} \in F\right\}
$$

the formal Laurent series field over $F$. By identifying $p(z) / q(z) \in F(z)$ with $p(z) q(z)^{-1} \in F\left(\left(z^{-1}\right)\right)$, where $p(z)$ and $q(z)(\neq 0)$ are polynomials, we view $F(z)$ as a subfield of $F\left(\left(z^{-1}\right)\right)$. We denote by $F^{m}, F[z]^{m}$ and $F\left(\left(z^{-1}\right)\right)^{m}$ the column vector space of dimension $m$ over $F, F[z]$ and $F\left(\left(z^{-1}\right)\right)$ respectively.

Definition 1 (order over $Z_{m} \times \mathbb{Z}$ ). For any two elements $(h, v)$ and $\left(h^{\prime}, v^{\prime}\right)$ in $Z_{m} \times \mathbb{Z}$, we define $(h, v)<\left(h^{\prime}, v^{\prime}\right)$ if $v<v^{\prime}$ or $v=v^{\prime}, h<h^{\prime}$.

The order defined above is linear [3]. It is clear that if $(j, n)<\left(j^{\prime}, n^{\prime}\right)$, then $n \leq n^{\prime}$ and $(j, n+x)<\left(j^{\prime}, n^{\prime}+x\right)$ for any $x \in \mathbb{Z}$.

For $1 \leq j \leq m$, we write $\underline{e}_{j}=(\stackrel{1}{0}, \ldots, 0, \stackrel{j}{1}, 0, \ldots, \stackrel{m}{0})^{\tau}$, which is the $j$ th standard base element in $F^{m}$, where $\tau$ means transpose; moreover, set

$$
z^{-n} \underline{e}_{j}=\left(\stackrel{1}{0}, \ldots, 0, z^{-n}, 0, \ldots, \stackrel{m}{0}\right)^{\tau} \in F\left(\left(z^{-1}\right)\right)^{m}, \quad \forall(j, n) \in Z_{m} \times \mathbb{Z},
$$

which is called the $(j, n)$ th monomial in $F\left(\left(z^{-1}\right)\right)^{m}$, and define

$$
\begin{aligned}
r z^{-n} \underline{e}_{j}=z^{-n} \underline{e}_{j} r=\left(\stackrel{1}{0}, \ldots, 0, r z^{j}, 0, \ldots, 0\right)^{\tau}, \\
\forall(j, n) \in Z_{m} \times \mathbb{Z}, r \in F\left(\left(z^{-1}\right)\right) .
\end{aligned}
$$

Definition 2. Any non-zero element $\underline{r}=\left(r_{1}, \ldots, r_{m}\right)^{\tau}$ in $F\left(\left(z^{-1}\right)\right)^{m}$, $r_{j}=\sum r_{j, n} z^{-n} \in F\left(\left(z^{-1}\right)\right)$, can be uniquely expressed as

$$
\underline{r}=\sum_{(i, t) \leq(j, n)} r_{j, n} z^{-n} \underline{e}_{j}, \quad r_{j, n} \in F,
$$

for some $(i, t) \in Z_{m} \times \mathbb{Z}$, which is called its monomial decomposition. $r_{j, n} z^{-n} \underline{e}_{j}$ is called the $(j, n)$ th term of $\underline{r} ; r_{j, n}$ the $(j, n)$ th coefficient of $\underline{r}$; $z^{-n} \underline{e}_{j}$ a monomial of $\underline{r}$ (written $z^{-n} \underline{e}_{j} \in \underline{r}$ ) if $r_{j, n} \neq 0$. For $\underline{0} \neq \underline{r} \in$ $F\left(\left(z^{-1}\right)\right)^{m}$, define

$$
\operatorname{Iv}(\underline{r})=\min \left\{(j, n) \mid r_{j, n} \neq 0,(j, n) \in Z_{m} \times \mathbb{Z}\right\} \in Z_{m} \times \mathbb{Z},
$$

and $\operatorname{Iv}(\underline{0})=(1, \infty)$. 
The pair $\operatorname{Iv}(\underline{r})$ is called the indexed valuation of $\underline{r} . \operatorname{If} \operatorname{Iv}(\underline{r})=(h, v)$, then $v$ is called the valuation of $\underline{r}$ and denoted by $v(\underline{r})$, and $h$ the index of $\underline{r}$ and denoted by $I(\underline{r})$; and $r_{h, v} z^{-v} \underline{e}_{h}$ is the leading term of $\underline{r}$, denoted by $\operatorname{Ld}(\underline{r})$.

It is clear that $v(\cdot)$ is the discrete valuation on $F\left(\left(z^{-1}\right)\right)$ when $m=1$. When $m>1$, we have

$v(\underline{r})=\min \left\{v\left(r_{j}\right) \mid 1 \leq j \leq m\right\}, \quad I(\underline{r})=\min \left\{j \mid v\left(r_{j}\right)=v(\underline{r}), 1 \leq j \leq m\right\}$.

Theorem 3. Let $\alpha, \beta \in F\left(\left(z^{-1}\right)\right)^{m}$.

(1) $\operatorname{Iv}(\alpha) \neq(1, \infty) \Leftrightarrow \alpha \neq 0$.

(2) If $\operatorname{Iv}(\alpha)=(h, v)$, then $\operatorname{Iv}(r \alpha)=(h, v+v(r))$ for any $0 \neq r \in$ $F\left(\left(z^{-1}\right)\right)$. In particular, $\operatorname{Iv}(r \alpha)=\operatorname{Iv}(\alpha)$ if $0 \neq r \in F$.

(3) $\operatorname{Iv}(\alpha+\beta) \geq \min \{\operatorname{Iv}(\alpha), \operatorname{Iv}(\beta)\}$, and equality holds if and only if $\operatorname{Ld}(\alpha)+\operatorname{Ld}(\beta) \neq \underline{0}$. In particular, $\operatorname{Iv}(\alpha+\beta)=\operatorname{Iv}(\alpha)$ if $\operatorname{Iv}(\alpha)<\operatorname{Iv}(\beta)$.

In studying the rational approximation problem of multi-formal Laurent series, we need the concept of limit with respect to the indexed valuation [15]. We say that a sequence $\left\{\underline{x}_{k}\right\}_{k \geq 0}$ in $F\left(\left(z^{-1}\right)\right)^{m}$ is convergent with respect to the indexed valuation if there exists an element $\underline{x} \in F\left(\left(z^{-1}\right)\right)^{m}$ (called a limit of $\left.\left\{\underline{x}_{k}\right\}_{k \geq 0}\right)$ which satisfies: for any $(h, v) \in Z_{m} \times \mathbb{Z}$ there is a positive integer $k_{0}$ such that $\operatorname{Iv}\left(\underline{x}_{k}-\underline{x}\right) \geq(h, v)$ whenever $k \geq k_{0}$.

One can verify that:

(1) If a sequence $\left\{\underline{x}_{k}\right\}_{k \geq 0}$ is convergent, then its limit $\underline{x} \in F\left(\left(z^{-1}\right)\right)^{m}$ is unique. Therefore we can write $\underline{x}=\lim _{k \rightarrow \infty} \underline{x}_{k}$.

(2) $F(z)^{m}$ is dense in $F\left(\left(z^{-1}\right)\right)^{m}$ in the sense that each element in $F\left(\left(z^{-1}\right)\right)^{m}$ is the limit of a sequence from $F(z)^{m}$.

\section{Optimal rational approximation}

DeFINITION 4. Let

$$
\frac{\underline{p}(z)}{q(z)}=\left(\frac{p_{1}(z)}{q(z)}, \ldots, \frac{p_{m}(z)}{q(z)}\right)^{\tau} \in F[z]^{m}
$$

be an $m$-tuple of rational fractions, where $q(z)$ is the common denominator of the $m$ components. The indexed valuation $\operatorname{Iv}(\underline{r}-p(z) / q(z))$ is called the precision of approximation of $\underline{r}$ by $p(z) / q(z)$. The tuple $p(z) / q(z)$ is called an optimal rational approximant to $\underline{r}$ if it satisfies the following two conditions:

- $\operatorname{Iv}\left(\underline{r}-\frac{\underline{u}(z)}{v(z)}\right)<\operatorname{Iv}\left(\underline{r}-\frac{\underline{p}(z)}{q(z)}\right) \forall \frac{\underline{u}(z)}{v(z)} \in F(z)^{m}, \operatorname{deg}(v(z))<\operatorname{deg}(q(z)) ;$

- $\operatorname{Iv}\left(\underline{r}-\frac{\underline{u}(z)}{v(z)}\right) \leq \operatorname{Iv}\left(\underline{r}-\frac{\underline{p}(z)}{q(z)}\right) \forall \frac{\underline{u}(z)}{v(z)} \in F(z)^{m}, \operatorname{deg}(v(z))=\operatorname{deg}(q(z))$. 
For a non-zero element $r=\sum_{i=0}^{t} b_{-i} z^{i}+\sum_{i \geq 1} b_{i} z^{-i}$ in $F\left(\left(z^{-1}\right)\right)$, where $t \geq 0$, define $\lfloor r\rfloor=\sum_{i=0}^{t} b_{-i} z^{i}$ and $\{r\}=\sum_{i \geq 1} b_{i} z^{-i}$, which are called the polynomial part and the remaining part of $r$, respectively [15].

For $\underline{r}=\left(\ldots, r_{j}(z), \ldots\right)^{\tau} \in F\left(\left(z^{-1}\right)\right)^{m}$, set $\lfloor\underline{r}\rfloor=\left(\ldots,\left\lfloor r_{j}(z)\right\rfloor, \ldots\right)^{\tau}$ and $\{\underline{r}\}=\left(\ldots,\left\{r_{j}(z)\right\}, \ldots\right)^{\tau}$. It is not difficult to see that $p(z) / q(z)$ is an optimal rational approximant to $\{\underline{r}\}$ of precision $(h, n)$ if and only if $\lfloor\underline{r}\rfloor+p(z) / q(z)$ is an optimal rational approximant to $\underline{r}$ of the same precision. Therefore, it is enough to consider elements $\underline{r}$ with positive valuation $(v(\underline{r})>0)$ in studying optimal rational approximation of formal Laurent series.

4. Multidimensional continued fraction algorithm. We denote by

$$
\operatorname{diag}\left(r_{1}, \ldots, r_{m}\right), \quad r_{j} \in F\left(\left(z^{-1}\right)\right),
$$

the diagonal matrix of order $m$ with the $j$ th diagonal element equal to $r_{j}$.

m-Continued Fraction Algorithm (m-CFA, for short). Given $\underline{r} \in$ $F\left(\left(z^{-1}\right)\right)^{m}$ with $\underline{r} \neq \underline{0}$ and $v(\underline{r})>0$, initially set $\underline{a}_{0}=\underline{0}, \Delta_{-1}=I_{m}=$ $\operatorname{diag}\left(\ldots, z^{-c_{0, j}}, \ldots\right), c_{0, j}=0$ for $1 \leq j \leq m$, and $\alpha_{0}=\underline{r}$. For any integer $k \geq 1$, suppose $\Delta_{k-2}=\operatorname{diag}\left(\ldots, z^{-c_{k-1, j}}, \ldots\right), c_{i, j} \in \mathbb{Z}$, and $\underline{0} \neq \alpha_{k-1}=$ $\left(\ldots, \alpha_{k-1, j}, \ldots\right)^{\tau} \in F\left(\left(z^{-1}\right)\right)^{m}$ have been obtained. Then the computations for the $k$ th round are defined by the following steps:

(1) Set $\left(h_{k}, c_{k}\right)=\operatorname{Iv}\left(\Delta_{k-2} \alpha_{k-1}\right)$.

(2) Set $\Delta_{k-1}=\operatorname{diag}\left(\ldots, z^{-c_{k, j}}, \ldots\right)$, which is an $m \times m$ diagonal matrix, where $c_{k, j}=c_{k-1, j}$ if $j \neq h_{k}$, and $c_{k, h_{k}}=c_{k}$.

(3) Set $\varrho_{k}=\left(\ldots, \varrho_{k, j}, \ldots\right)^{\tau} \in F\left(\left(z^{-1}\right)\right)^{m}$, where $\varrho_{k, j}=\alpha_{k-1, j} / \alpha_{k-1, h_{k}}$ if $j \neq h_{k}$, and $\varrho_{k, h_{k}}=1 / \alpha_{k-1, h_{k}}$.

(4) Set $\alpha_{k}=\left\{\varrho_{k}\right\}$ and $\underline{a}_{k}=\left\lfloor\varrho_{k}\right\rfloor$. If $\alpha_{k}=\underline{0}$, then set $\mu=k$, and the algorithm terminates.

Define $\mu=\infty$ if the above procedure never terminates.

By letting $\mathrm{m}$-CFA act on $\underline{r}$, we get an expansion of the form

$$
C(\underline{r})=\left[\underline{0}, h_{1}, \underline{a}_{1}, \ldots, h_{k}, \underline{a}_{k}, \ldots\right], \quad 1 \leq k \leq \mu .
$$

We call $C(\underline{r})$ the multi-continued fraction expansion of $\underline{r}$, and $\mu$ the length of $C(\underline{r})$.

In what follows we keep the notation $C(\underline{r})$ and all the notations appearing in the process of generating $C(\underline{r})$, and define

$$
\underline{a}_{k}=\left(a_{k, 1}, \ldots, a_{k, j}, \ldots, a_{k, m}\right) .
$$

For the case $\mu<\infty$, we see that $\alpha_{\mu}=\underline{0}$, and it is convenient to set

$$
\left(h_{\mu+1}, c_{\mu+1}\right)=\operatorname{Iv}\left(\Delta_{\mu} \alpha_{\mu}\right)=(1, \infty) .
$$

TheOREM 5. $\alpha_{k-1, h_{k}} \neq 0$ for $1 \leq k \leq \mu$. As a consequence, the $m$-CFA is well defined, and $0 \neq a_{k-1, h_{k}} \in F[z], \operatorname{deg}\left(a_{k-1, h_{k}}\right) \geq 1$ for $1 \leq k \leq \mu$. 
Proof. From $\left(h_{k}, c_{k}\right)=\operatorname{Iv}\left(\Delta_{k-2} \alpha_{k-1}\right)$, we see that $c_{k-1, h_{k}}+v\left(\alpha_{k-1, h_{k}}\right)$ $=c_{k}$, thus, $v\left(\alpha_{k-1, h_{k}}\right)=-c_{k-1, h_{k}}+c_{k} \in \mathbb{Z}$, hence $\alpha_{k-1, h_{k}} \neq 0$.

REMARK. When $m=1$, the m-CFA is exactly the classical continued fraction algorithm [14] for formal power series. In fact, when $m=1$, we have $h_{k}=1$ for all $k$, hence both step (1) and step (2) at each round are unnecessary. Now, the 1-CFA is as follows (we write $\underline{r}=r, \underline{a}_{k}=a_{k}$ ): Initially, set $a_{0}=0, \alpha_{0}=r$. For any integer $k \geq 1$, suppose $\left[a_{0}, a_{1}, \ldots, a_{k-1}\right]$ and $0 \neq \alpha_{k-1} \in F\left(\left(z^{-1}\right)\right)$ have been obtained. Then the computations for the $k$ th round are defined by the following steps:

(1) Set $\varrho_{k}=1 / \alpha_{k-1}$.

(2) Set $\alpha_{k}=\left\{\varrho_{k}\right\}$ and $a_{k}=\left\lfloor\varrho_{k}\right\rfloor$. If $\alpha_{k}=\underline{0}$, then set $\mu=k$, and the algorithm terminates.

Define $\mu=\infty$ if the above procedure never terminates.

5. Three conditions satisfied by the multi-continued fraction expansion $C(\underline{r})$. Define

$$
\left\{\begin{array}{l}
t_{0}=0 \\
t_{k}=\operatorname{deg}\left(a_{k, h_{k}}(z)\right), \quad 1 \leq k \leq \mu, \\
v_{0, j}=0 \\
v_{k, j}=\sum_{h_{i}=j, 1 \leq i \leq k} t_{i}, \quad 1 \leq k \leq \mu, 1 \leq j \leq m, \\
v_{k}=v_{k, h_{k}}, \quad 1 \leq k \leq \mu, \\
D_{k}=\operatorname{diag}\left(z^{-v_{k, 1}}, \ldots, z^{-v_{k, m}}\right), \quad 0 \leq k \leq \mu, \\
t_{\mu}=\infty \\
\left(h_{\mu+1}, v_{\mu+1}\right)=(1, \infty) \quad \text { if } \mu<\infty
\end{array}\right.
$$

Theorem 6. For $1 \leq k \leq \mu, C(\underline{r})$ satisfies:

- Condition 1: $t_{k} \geq 1$,

- Condition 2: $\operatorname{Iv}\left(D_{k} \underline{a}_{k}\right)=\left(h_{k}, v_{k-1, h_{k}}\right)$,

- Condition 3: $\left(h_{k}, v_{k-1, h_{k}}\right)<\left(h_{k+1}, v_{k+1}\right)$.

Before proving Theorem 6 we make some preparations. In particular, we introduce the concept of a D-matrix.

Definition 7. We call a diagonal matrix over $F\left(\left(z^{-1}\right)\right)$ a $D$-matrix if each of its diagonal elements is a power of $z$.

It is clear that both $D_{k}$ and $\Delta_{k-1}$ are D-matrices.

Lemma 8. Let $\underline{0} \neq \varrho \in F\left(\left(z^{-1}\right)\right)^{m}$ and $I(\Delta \varrho)=h$, where $\Delta$ is a $D$ matrix. Then

$$
\operatorname{Iv}(\Delta \varrho)= \begin{cases}\operatorname{Iv}(\Delta\lfloor\varrho\rfloor)<\operatorname{Iv}(\Delta\{\varrho\}) & \text { if }\left\lfloor\varrho_{h}\right\rfloor \neq 0, \\ \operatorname{Iv}(\Delta\{\varrho\})<\operatorname{Iv}(\Delta\lfloor\varrho\rfloor) & \text { if }\left\lfloor\varrho_{h}\right\rfloor=0,\end{cases}
$$

where $\varrho_{h}$ is the hth component of $\varrho$. 
Proof. Set $\Delta=\operatorname{diag}\left(\ldots, z^{-b_{j}}, \ldots\right)$. Then $\operatorname{Iv}(\Delta \varrho)=\operatorname{Iv}\left(z^{-b_{h}} z^{-v\left(\varrho_{h}\right)} \underline{e}_{h}\right)$. Noting that there are no common monomials in $\Delta\{\varrho\}$ and $\Delta\lfloor\varrho\rfloor$, we see that $\operatorname{Iv}(\Delta\{\varrho\}) \neq \operatorname{Iv}(\Delta\lfloor\varrho\rfloor)$, and then $\operatorname{Iv}(\Delta \varrho)=\min \{\operatorname{Iv}(\Delta\lfloor\varrho\rfloor), \operatorname{Iv}(\Delta\{\varrho\})\}$, which leads to the result by observing that $\operatorname{Iv}(\Delta \varrho)=\operatorname{Iv}(\Delta\lfloor\varrho\rfloor)<\operatorname{Iv}(\Delta\{\varrho\})$ if and only if $z^{-v\left(\varrho_{h}\right)} \underline{e}_{h} \in\lfloor\varrho\rfloor$, and the latter holds true if and only if $\left\lfloor\varrho_{h}\right\rfloor \neq 0$.

LEMMa 9.

(1) $t_{k}=\operatorname{deg}\left(a_{k, h_{k}}\right)=-v\left(\varrho_{k, h_{k}}\right)=v\left(\alpha_{k-1, h_{k}}\right)>0$ for $1 \leq k \leq \mu$.

(2) $v_{k, j}=c_{k, j}$ and $v_{k}=c_{k}$ for $0 \leq k \leq \mu$ and $1 \leq j \leq m$. As a consequence, $D_{k}=\Delta_{k-1}$ for $0 \leq k \leq \mu$.

(3) $\operatorname{Iv}\left(\Delta_{k-1} \varrho_{k}\right)=\left(h_{k}, v_{k-1, h_{k}}\right)$.

Proof. (1) Noting that $\alpha_{k-1, h_{k}} \neq 0$ and $\alpha_{k-1}$ is the remaining part of $\varrho_{k-1}$, we see that $0<v\left(\alpha_{k-1, h_{k}}\right) \neq \infty$. Since $a_{k, h_{k}}=\left\lfloor\varrho_{k, h_{k}}\right\rfloor=\left\lfloor\alpha_{k-1, h_{k}}^{-1}\right\rfloor$, we obtain

$$
\begin{aligned}
t_{k} & =\operatorname{deg}\left(a_{k, h_{k}}\right)=-v\left(\left\lfloor\varrho_{k, h_{k}}\right\rfloor\right)=-v\left(\left\lfloor\alpha_{k-1, h_{k}}^{-1}\right\rfloor\right) \\
& =-v\left(\alpha_{k-1, h_{k}}^{-1}\right)=v\left(\alpha_{k-1, h_{k}}\right)>0 .
\end{aligned}
$$

(2) By definition,

$$
v_{k, j}= \begin{cases}v_{k-1, j} & \text { if } j \neq h_{k}, \\ v_{k}=v_{k-1, h_{k}}+t_{k} & \text { if } j=h_{k} .\end{cases}
$$

From $\operatorname{Iv}\left(\Delta_{k-2} \alpha_{k-1}\right)=\left(h_{k}, c_{k}\right)$, we see that $c_{k}=c_{k-1, h_{k}}+v\left(\alpha_{k-1, h_{k}}\right)=$ $c_{k-1, h_{k}}+t_{k}$, so

$$
c_{k, j}= \begin{cases}c_{k-1, j} & \text { if } j \neq h_{k}, \\ c_{k}=c_{k-1, h_{k}}+t_{k} & \text { if } j=h_{k} .\end{cases}
$$

Therefore, the $v_{k, j}$ satisfy the same recurrence relation as $c_{k, j}$, and they have the same initial values: $v_{0, j}=c_{0, j}$, so $v_{k, j}=c_{k, j}$ and $v_{k}=v_{k, h_{k}}=c_{k, h_{k}}=c_{k}$.

(3) From (7) we see that

$$
\Delta_{k-1}=\left(\begin{array}{ccc}
I_{h_{k}-1} & 0 & 0 \\
0 & z^{-t_{k}} & 0 \\
0 & 0 & I_{m-h_{k}}
\end{array}\right) \Delta_{k-2}
$$

and

$$
\varrho_{k}=\left(\begin{array}{ccc}
I_{h_{k}-1} & 0 & 0 \\
0 & \alpha_{k-1, h_{k}}^{-1} & 0 \\
0 & 0 & I_{m-h_{k}}
\end{array}\right) \alpha_{k-1} \alpha_{k-1, h_{k}}^{-1}
$$

Then

$$
\Delta_{k-1} \varrho_{k}=\left(\begin{array}{ccc}
I_{h_{k}-1} & 0 & 0 \\
0 & z^{-t_{k}} \alpha_{k-1, h_{k}}^{-1} & 0 \\
0 & 0 & I_{m-h_{k}}
\end{array}\right) \Delta_{k-2} \alpha_{k-1} \alpha_{k-1, h_{k}}^{-1}
$$


Since $v\left(z^{-t_{k}} \alpha_{k-1, h_{k}}^{-1}\right)=0$, we obtain

$v\left(\left(\begin{array}{ccc}I_{h_{k}-1} & 0 & 0 \\ 0 & z^{-t_{k}} \alpha_{k-1, h_{k}}^{-1} & 0 \\ 0 & 0 & I_{m-h_{k}}\end{array}\right) \Delta_{k-2} \alpha_{k-1}\right)=v\left(\Delta_{k-2} \alpha_{k-1}\right)=\left(h_{k}, c_{k}\right)$

Thus

$$
\operatorname{Iv}\left(\Delta_{k-1} \varrho_{k}\right)=\left(h_{k}, c_{k}-v\left(\alpha_{k-1, h_{k}}\right)\right)=\left(h_{k}, v_{k-1, h_{k}}\right) .
$$

Proof of Theorem 6. From Lemma 9 we see that Condition 1 holds true. Noting that $h_{k}=I\left(\Delta_{k-1} \varrho_{k}\right)$ and $\left\lfloor\varrho_{k, h_{k}}\right\rfloor \neq 0$ (see Lemma 9), from Lemma 8 we get

$$
\operatorname{Iv}\left(\Delta_{k-1} \varrho_{k}\right)=\operatorname{Iv}\left(\Delta_{k-1}\left\lfloor\varrho_{k}\right\rfloor\right)<\operatorname{Iv}\left(\Delta_{k-1}\left\{\varrho_{k}\right\}\right) .
$$

Since $\underline{a}_{k}=\left\lfloor\varrho_{k}\right\rfloor, \alpha_{k}=\left\{\varrho_{k}\right\}$ and $\operatorname{Iv}\left(\Delta_{k-1} \varrho_{k}\right)=\left(h_{k}, v_{k-1, h_{k}}\right)$, we get

$$
\left(h_{k}, v_{k-1, h_{k}}\right)=\operatorname{Iv}\left(\Delta_{k-1} \underline{a}_{k}\right)<\operatorname{Iv}\left(\Delta_{k-1} \alpha_{k}\right)=\left(h_{k+1}, c_{k+1}\right)=\left(h_{k+1}, v_{k+1}\right),
$$

which together with $\Delta_{k-1}=D_{k}$ tells us that $C(\underline{r})$ satisfies Conditions 2 and 3 .

6. m-CFA and optimal rational approximations. In this section we show how $C(\underline{r})$ provides optimal rational approximations to $\underline{r}$ by rational fractions $\left(\frac{p}{q}_{k}\right), 0 \leq k \leq \mu$, defined below.

Define iteratively the square matrices $B_{k}$ of order $m+1$ over $F[z]$ :

$$
\left\{\begin{array}{l}
B_{0}=I_{m+1}, \\
B_{k}=B_{k-1} E_{h_{k}} A\left(\underline{a}_{k}\right), \quad 1 \leq k \leq \mu,
\end{array}\right.
$$

where

$$
\left\{\begin{array}{l}
E_{h}=\left(\underline{e}_{1} \underline{e}_{2} \cdots \underline{e}_{h-1} \underline{e}_{m+1} \underline{e}_{h+1} \cdots \underline{e}_{m} \underline{e}_{h}\right), \\
A\left(\underline{a}_{k}\right)=\left(\begin{array}{cc}
I_{m} & \underline{a}_{k} \\
0 & 1
\end{array}\right) .
\end{array}\right.
$$

In other words, $E_{h}$ is the matrix of order $m+1$ obtained by exchanging the $h$ th and $(m+1)$ th columns of the identity matrix $I_{m+1}$.

Define

$$
\left(\begin{array}{l}
\underline{p}_{k} \\
q_{k}
\end{array}\right)=B_{k}(0 \ldots 01)^{\tau},
$$

which is the last column of $B_{k}$, where $\underline{p}_{k}(z) \in F[z]^{m}$ and $q_{k}(z) \in F[z]$.

REMARK. When $m=1$, write $\underline{p}_{k}=p_{k} \in F[z], \underline{a}_{k}=a_{k} \in F[z]$; we claim that $p_{k}$ and $q_{k}$ satisfy the following recurrence relation:

$$
\left\{\begin{array}{l}
p_{k}=p_{k-2}+a_{k} p_{k-1}, \\
q_{k}=q_{k-2}+a_{k} q_{k-1},
\end{array} \quad \text { for } k \geq 1,\right.
$$


where $\left(p_{-1}, q_{-1}\right)=(1,0)$ and $\left(p_{0}, q_{0}\right)=(0,1)$, hence the rational fractions $\left(\begin{array}{l}p_{k} \\ q_{k}\end{array}\right)$ are exactly the rational approximants provided by the classical continued fraction algorithm [14]. In fact, we can prove (11) and

$$
B_{k}=\left(\begin{array}{cc}
p_{k-1} & p_{k} \\
q_{k-1} & q_{k}
\end{array}\right), \quad k \geq 0,
$$

together by induction on $k$. It is easy to check (12) for $k=0$. Assume

$$
B_{k-1}=\left(\begin{array}{cc}
p_{k-2} & p_{k-1} \\
q_{k-2} & q_{k-1}
\end{array}\right)
$$

then the first column of $B_{k}$ is

$$
B_{k}\left(\begin{array}{ll}
1 & 0
\end{array}\right)^{\tau}=B_{k-1} E_{1} A\left(a_{k}\right)\left(\begin{array}{lll}
1 & 0
\end{array}\right)^{\tau}=B_{k-1} E_{1}\left(\begin{array}{ll}
1 & 0
\end{array}\right)^{\tau}=B_{k-1}\left(\begin{array}{ll}
0 & 1
\end{array}\right)^{\tau}=\left(\begin{array}{l}
p_{k-1} \\
q_{k-1}
\end{array}\right),
$$

hence (12) is true because $\left(\begin{array}{l}p_{k} \\ q_{k}\end{array}\right)$ is the second column of $B_{k}$ by definition. Then we have

$$
\left(\begin{array}{c}
p_{k} \\
q_{k}
\end{array}\right)=B_{k}\left(\begin{array}{ll}
0 & 1
\end{array}\right)^{\tau}=B_{k-1} E_{1} A\left(a_{k}\right)\left(\begin{array}{ll}
0 & 1
\end{array}\right)^{\tau}=B_{k-1}\left(\begin{array}{c}
1 \\
a_{k}
\end{array}\right)=\left(\begin{array}{c}
p_{k-2}+a_{k} p_{k-1} \\
q_{k-2}+a_{k} q_{k-1}
\end{array}\right),
$$

hence (11) holds true for $k$.

Define

$$
\left\{\begin{array}{l}
d_{0}=0, \\
d_{k}=\sum_{1 \leq i \leq k} t_{i}, \\
n_{k}=d_{k-1}+v_{k}, \\
d_{\mu+1}=t_{\mu+1}=n_{\mu+1}=\infty \text { if } \mu<\infty .
\end{array}\right.
$$

From the fact that $n_{k}=d_{k-1}+v_{k}=d_{k}+v_{k-1, h_{k}}$ and $n_{k+1}=d_{k}+v_{k+1}$, we see immediately that Condition 3: $\left(h_{k}, v_{k-1, h_{k}}\right)<\left(h_{k+1}, v_{k+1}\right) \forall 1 \leq k \leq \mu$, is equivalent to the following condition:

$$
\left(h_{k}, n_{k}\right)<\left(h_{k+1}, n_{k+1}\right) \quad \forall 1 \leq k \leq \mu .
$$

THEOREM 10.

(1) $\operatorname{gcd}\left(q_{k}(z), \ldots, p_{k, j}(z), \ldots\right)=1$ for all $0 \leq k \leq \mu$, where $p_{k, j}(z)$ is the jth component of $\underline{p}_{k}(z)$.

(2) $\operatorname{deg}\left(q_{k}(z)\right)=d_{k}$ for all $0 \leq k \leq \mu$.

TheOREM 11. $\operatorname{Iv}\left(\underline{r}-\underline{p}_{k}(z) / q_{k}(z)\right)=\left(h_{k+1}, n_{k+1}\right)$. As a consequence,

$$
\underline{r}= \begin{cases}\frac{\underline{p}_{\mu}(z)}{q_{\mu}(z)} & \text { if } \mu<\infty, \\ \lim _{k \rightarrow \infty} \frac{p_{k}(z)}{q_{k}(z)} & \text { if } \mu=\infty .\end{cases}
$$


We call the rational fraction $\underline{p}_{k}(z) / q_{k}(z)(0 \leq k \leq \mu)$ the $k$ th rational approximant of $C(\underline{r})$, and we say $C(\underline{r})$ converges to $\underline{r}$ in the sense that (15) holds. The following theorem shows that $C(\underline{r})$ provides optimal rational approximations to $\underline{r}$.

Theorem 12. Assume $q(z) \in F[z], d_{k} \leq \operatorname{deg}(q(z))<d_{k+1}$ and $\underline{p}(z) \in$ $F[z]^{m}$ for some $0 \leq k \leq \mu$. Then

$$
\operatorname{Iv}\left(\underline{r}-\frac{\underline{p}(z)}{q(z)}\right) \leq \operatorname{Iv}\left(\underline{r}-\frac{\underline{p}_{k}(z)}{q_{k}(z)}\right)=\left(h_{k+1}, n_{k+1}\right) .
$$

As a consequence, no $\underline{p}(z) / q(z)$ with $\operatorname{deg}(q(z))<d_{k+1}$ approximates $\underline{r}$ better than $\underline{p}_{k}(z) / q_{k}(z)$. In particular:

(1) Each $\underline{p}_{k}(z) / q_{k}(z), 0 \leq k \leq \mu$, is an optimal rational approximant to $\underline{r}$.

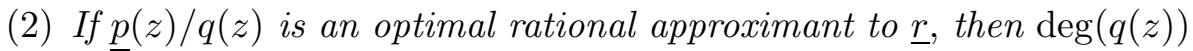
$=\bar{d}_{k}$ for some $k, 0 \leq k \leq \mu$.

\section{Proof of the theorems}

7.1. Proof of Theorem 10. First we express $q_{k}(z)$ explicitly. To do this, for $0 \leq k \leq \mu$ we denote by $P_{k-1}$ the $m \times m$ submatrix of $B_{k}$ which is made up of the first $m$ columns and the first $m$ rows, and by $Q_{k-1}$ the $1 \times m$ submatrix of $B_{k}$ made up of the first $m$ columns and the last row; moreover, denote by $\underline{P}_{k-1, j}\left(\in F[z]^{m}\right)$ the $j$ th column of $P_{k-1}$, and by $Q_{k-1, j}(\in F[z])$ the $j$ th component of $Q_{k-1}$ for $1 \leq j \leq m$.

Lemma 13. For $1 \leq k \leq \mu$, we have:

$$
\begin{aligned}
B_{k-1} E_{h_{k}} & =\left(\begin{array}{ll}
P_{k-1} & P_{k-2, h_{k}} \\
Q_{k-1} & Q_{k-2, h_{k}}
\end{array}\right) . \\
\left(\begin{array}{c}
P_{k-1, j} \\
Q_{k-1, j}
\end{array}\right) & =\left\{\begin{array}{l}
\left(\begin{array}{l}
P_{k-2, j} \\
Q_{k-2, j}
\end{array}\right) \text { if } j \neq h_{k}, \\
\left(\begin{array}{l}
\underline{p}_{k-1} \\
q_{k-1}
\end{array}\right) \quad \text { if } j=h_{k} .
\end{array}\right.
\end{aligned}
$$

Proof. (1) We have

$$
B_{k-1} E_{h_{k}}\left(\begin{array}{c}
I_{m} \\
0
\end{array}\right)=B_{k-1} E_{h_{k}} A\left(\underline{a}_{k}\right)\left(\begin{array}{c}
I_{m} \\
0
\end{array}\right)=B_{k}\left(\begin{array}{c}
I_{m} \\
0
\end{array}\right)=\left(\begin{array}{c}
P_{k-1} \\
Q_{k-1}
\end{array}\right),
$$

and

$$
B_{k-1} E_{h_{k}}(\underline{\underline{0}} 1)=B_{k-1} \underline{e}_{h_{k}}=\left(\begin{array}{c}
P_{k-2, h_{k}} \\
Q_{k-2, h_{k}}
\end{array}\right) .
$$

Hence, we get (1). 
(2) We have

$$
\left(\begin{array}{c}
P_{k-1, j} \\
Q_{k-1, j}
\end{array}\right)=B_{k-1} E_{h_{k}} \underline{e}_{j}=\left\{\begin{array}{l}
B_{k-1} \underline{e}_{j}=\left(\begin{array}{c}
P_{k-2, j} \\
Q_{k-2, j}
\end{array}\right) \quad \text { if } j \neq h_{k}, \\
B_{k-1} \underline{e}_{m+1}=\left(\begin{array}{l}
\underline{p}_{k-1} \\
q_{k-1}
\end{array}\right) \text { if } j=h_{k} .
\end{array}\right.
$$

Now, for $k \geq 1, q_{k}$ can be expressed explicitly as

$$
\begin{aligned}
q_{k}(z) & \\
& =\left(\begin{array}{ll}
0_{1 \times m} & 1
\end{array}\right) B_{k}\left(\begin{array}{c}
\underline{0} \\
1
\end{array}\right)=\left(\begin{array}{ll}
0_{1 \times m} & 1
\end{array}\right) B_{k-1} E_{h_{k}}\left(\begin{array}{cc}
I_{m} & \underline{a}_{k} \\
\underline{0} & 1
\end{array}\right)\left(\begin{array}{c}
\underline{0} \\
1
\end{array}\right) \\
& =\left(\begin{array}{ll}
0_{1 \times m} & 1
\end{array}\right)\left(\begin{array}{cc}
P_{k-1} & P_{k-2, h_{k}} \\
Q_{k-1} & Q_{k-2, h_{k}}
\end{array}\right)\left(\begin{array}{c}
\underline{a}_{k} \\
1
\end{array}\right)=Q_{k-1} \underline{a}_{k}(z)+Q_{k-2, h_{k}} \\
& =q_{k-1}(z) a_{k, h_{k}}(z)+\sum_{j \neq h_{k}, Q_{k-1, j} \neq 0,1 \leq j \leq m} Q_{k-1, j} a_{k, j}(z)+Q_{k-2, h_{k}} .
\end{aligned}
$$

To evaluate the degree of $a_{k, j}(z)$ and to show how $Q_{k-1, j}$ depends on some $q_{i}(z)(0 \leq i \leq k-1)$, we define a function $l(k, j)$, which is associated to $C(\underline{r})$ and defined on the set $[1, \mu] \times Z_{m}([1, \mu]=\{k \in \mathbb{Z} \mid 1 \leq k \leq \mu\})$, in the following way: $l(k, j)=k_{0}$ if there exists an integer $k_{0}$ such that $1 \leq k_{0} \leq k$, $h_{k_{0}}=j$ and $h_{i} \neq j$ for all $k_{0}<i \leq k$; and $l(k, j)=0$ otherwise. It is clear that

$$
\left\{\begin{array}{l}
l\left(k, h_{k}\right)=k \\
l(k, j)<k \text { if } j \neq h_{k} \\
h_{l(k, j)}=j, \\
v_{k, j}=v_{l(k, j)} .
\end{array}\right.
$$

Lemma 14. For $1 \leq k \leq \mu$, we have

$$
\left(\begin{array}{c}
P_{k-1, j} \\
Q_{k-1, j}
\end{array}\right)= \begin{cases}\left(\begin{array}{l}
\underline{p}_{l(k, j)-1} \\
q_{l(k, j)-1}
\end{array}\right) & \text { if } l(k, j) \geq 1, \\
\left(\begin{array}{c}
\underline{e}_{j} \\
0
\end{array}\right) & \text { if } l(k, j)=0 .\end{cases}
$$

As a consequence, $l(k, j) \geq 1$ if $Q_{k-1, j} \neq 0$.

(2) For $j \neq h_{k}$,

$$
\begin{cases}\operatorname{deg}\left(a_{k, j}(z)\right)<d_{k}-d_{l(k, j)-1} & \text { if } l(k, j) \geq 1 \\ a_{k, j}(z) \in F & \text { if } l(k, j)=0\end{cases}
$$


Proof. (1) For $j=h_{k}$, we have seen

$$
\left(\begin{array}{c}
P_{k-1, h_{k}} \\
Q_{k-1, h_{k}}
\end{array}\right)=\left(\begin{array}{c}
\underline{p}_{k-1} \\
q_{k-1}
\end{array}\right)=\left(\begin{array}{l}
\underline{p}_{l\left(k, h_{k}\right)-1} \\
q_{l\left(k, h_{k}\right)-1}
\end{array}\right) .
$$

For $j \neq h_{k}$ we have

$$
\begin{aligned}
& \left(\begin{array}{c}
P_{k-1, j} \\
Q_{k-1, j}
\end{array}\right)=\left(\begin{array}{c}
P_{k-2, j} \\
Q_{k-2, j}
\end{array}\right)=\ldots
\end{aligned}
$$

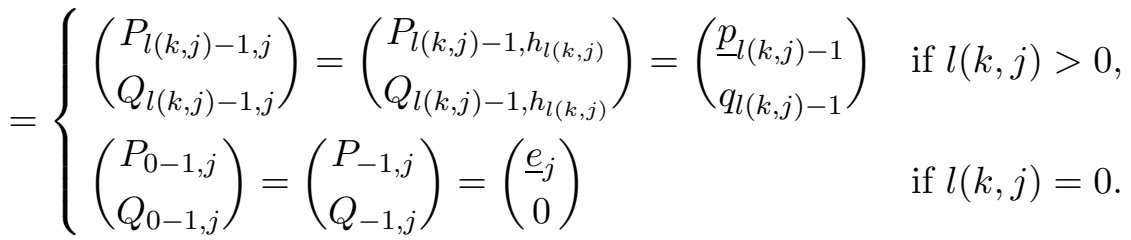

(2) From $D_{k} \underline{a}_{k}=\sum_{1<j<m} z^{-v_{k, j}} a_{k, j} \underline{e}_{j}$ and $\operatorname{Iv}\left(D_{k} \underline{a}_{k}\right)=\left(h_{k}, v_{k-1, h_{k}}\right)$ and the assumption $j \neq h_{k}$, we see that

$$
\left(j, v_{k, j}-\operatorname{deg}\left(a_{k, j}\right)\right)=\operatorname{Iv}\left(z^{-v_{k, j}} a_{k, j} \underline{e}_{j}\right)>\operatorname{Iv}\left(D_{k} \underline{a}_{k}\right)=\left(h_{k}, v_{k-1, h_{k}}\right),
$$

and then

$$
v_{k, j}-\operatorname{deg}\left(a_{k, j}\right) \geq v_{k-1, h_{k}} .
$$

If $l(k, j)>0$, from $(18)$ we get

$$
\begin{aligned}
\left(j, d_{k}+v_{k, j}-\operatorname{deg}\left(a_{k, j}\right)\right) & >\left(h_{k}, d_{k}+v_{k-1, h_{k}}\right)=\left(h_{k}, n_{k}\right) \\
& >\left(h_{l(k, j)}, n_{l(k, j)}\right)=\left(j, n_{l(k, j)}\right),
\end{aligned}
$$

so

$$
d_{k}+v_{k, j}-\operatorname{deg}\left(a_{k, j}\right)>n_{l(k, j)}=d_{l(k, j)-1}+v_{l(k, j)}=d_{l(k, j)-1}+v_{k, j},
$$

hence $\operatorname{deg}\left(a_{k, j}\right)<d_{k}-d_{l(k, j)-1}$. If $l(k, j)=0$, then $v_{k, j}=0$, and from (19) we have $\operatorname{deg}\left(a_{k, j}\right) \leq v_{k, j}-v_{k-1, h_{k}}=-v_{k-1, h_{k}} \leq 0$, hence $\operatorname{deg}\left(a_{k, j}\right) \leq 0$, i.e., $a_{k, j}(z) \in F$.

Now we turn to the proof of Theorem 10.

(1) By definition, $B_{k}$ is a matrix over $F[z]$ and $\operatorname{det}\left(B_{k}\right)=1$, which leads to assertion (1).

(2) We argue by induction on $k$. For $k=0$, we have $q_{0}(z)=1$, hence $\operatorname{deg}\left(q_{0}\right)=0=d_{0}$. Assume $\operatorname{deg}\left(q_{i}\right)=d_{i}$ for $i<k$ and $k \geq 1$. From (16) and Lemma 14 we see that

$$
q_{k}(z)=q_{k-1}(z) a_{k, h_{k}}(z)+\sum_{j \neq h_{k}, l(k, j) \geq 1,1 \leq j \leq m} q_{l(k, j)-1, j} a_{k, j}(z)+Q_{k-2, h_{k}} .
$$

The required result $\operatorname{deg}\left(q_{k}(z)\right)=d_{k}$ follows by observing the following facts:

- $\operatorname{deg}\left(q_{k-1}(z) a_{k, h_{k}}(z)\right)=d_{k-1}+t_{k}=d_{k}$ (induction assumption). 
- For $j \neq h_{k}$ and $l(k, j) \geq 1$, we see that (by induction assumption)

$$
\operatorname{deg}\left(q_{l(k, j)-1} a_{k, j}(z)\right)<d_{l(k, j)-1}+d_{k}-d_{l(k, j)-1}=d_{k} .
$$

- If $Q_{k-2, h_{k}} \neq 0$, then $l\left(k-1, h_{k}\right) \geq 1$, and hence $Q_{k-2, h_{k}}=q_{l\left(k-1, h_{k}\right)-1}$. So, $\operatorname{deg}\left(Q_{k-2, h_{k}}\right)=\operatorname{deg}\left(q_{l\left(k-1, h_{k}\right)-1}\right)=d_{l\left(k-1, h_{k}\right)-1}<d_{k}$.

7.2. Proof of Theorem 11. For $0 \leq k \leq \mu$ we define

$$
\begin{aligned}
\underline{r}_{k} & =\underline{r} q_{k}-\underline{p}_{k}, \\
-R_{k-1} & =\underline{r} Q_{k-1}-P_{k-1} .
\end{aligned}
$$

We call $\underline{r}_{k}$ the $k$ th remainder vector, and $R_{k-1}$ the $(k-1)$ th remainder matrix.

Theorem 11 is an easy consequence of the following

Proposition 15. For $1 \leq k \leq \mu$, we have

(1) $R_{k-1} \varrho_{k}=-R_{k-2, h_{k}}$ and $\underline{r}_{k}=R_{k-1} \alpha_{k}$.

(2) $\operatorname{Iv}\left(R_{k-1} \alpha_{k}\right)=\operatorname{Iv}\left(D_{k} \alpha_{k}\right)$.

(3) $\operatorname{Iv}\left(\underline{r}_{k}\right)=\left(h_{k+1}, v_{k+1}\right)$.

Proposition 15 will be proved later, now we prove Theorem 11 based on it. From item (3) of Proposition 15 we get immediately

$$
\operatorname{Iv}\left(\underline{r}-\frac{\underline{p}_{k}(z)}{q_{k}(z)}\right)=\operatorname{Iv}\left(\frac{\underline{r}_{k}}{q_{k}(z)}\right)=\left(h_{k+1}, v_{k+1}+d_{k}\right)=\left(h_{k+1}, n_{k+1}\right) .
$$

To prove Proposition 15, we denote by $R_{k-1, j}$ the $j$ th column of $R_{k-1}$. It is clear that

$$
\begin{aligned}
& \left(-I_{m}, \underline{r}\right) B_{k}=\left(-R_{k-1}, \underline{r}_{k}\right), \\
& \left(-I_{m}, \underline{r}\right)\left(\begin{array}{c}
P_{k-1, j} \\
Q_{k-1, j}
\end{array}\right)=-R_{k-1, j} .
\end{aligned}
$$

Lemma 16. For $1 \leq k \leq \mu$, we have

(2) $-R_{k-1, j}= \begin{cases}-R_{k-2, j} & \text { if } j \neq h_{k}, \\ \underline{r}_{k-1} & \text { if } j=h_{k} .\end{cases}$

Proof. (1) We have

$$
\left(-I_{m}, \underline{r}\right) B_{k-1} E_{h_{k}}=\left(-I_{m}, \underline{r}\right)\left(\begin{array}{cc}
P_{k-1} & P_{k-2, h_{k}} \\
Q_{k-1} & Q_{k-1, h_{k}}
\end{array}\right)=\left(-R_{k-1},-R_{k-2, h_{k}}\right) .
$$


(2) By (23),

$$
\begin{aligned}
-R_{k-1, j}= & \left(-I_{m}, \underline{r}\right)\left(\begin{array}{l}
P_{k-1, j} \\
Q_{k-1, j}
\end{array}\right) \\
= & \begin{cases}\left(-I_{m}, \underline{r}\right)\left(\begin{array}{l}
P_{k-2, j} \\
Q_{k-2, j}
\end{array}\right)=-R_{k-2, j} & \text { if } j \neq h_{k}, \\
\left(-I_{m}, \underline{r}\right)\left(\begin{array}{l}
\underline{p}_{k-1} \\
q_{k-1}
\end{array}\right)=\underline{r}_{k-1} & \text { if } j=h_{k} .\end{cases}
\end{aligned}
$$

Proof of Proposition 15(1). We argue by induction on $k$. It is easy to check $\underline{r}_{0}=R_{-1} \alpha_{0}$. Now assume $\underline{r}_{k-1}=R_{k-2} \alpha_{k-1}$. We have

$$
\begin{aligned}
& -R_{k-1} \varrho_{k}-R_{k-2, h_{k}}=\left(-R_{k-1},-R_{k-2, h_{k}}\right)\left(\begin{array}{c}
\varrho_{k} \\
1
\end{array}\right) \\
& =\left(-I_{m}, \underline{r}\right) B_{k-1} E_{h_{k}}\left(\begin{array}{c}
\varrho_{k} \\
1
\end{array}\right)=\left(-I_{m}, \underline{r}\right) B_{k-1} E_{h_{k}} E_{h_{k}}\left(\begin{array}{c}
\alpha_{k-1} \\
1
\end{array}\right) \alpha_{k-1, h_{k}}^{-1} \\
& =\left(-R_{k-2}, \underline{r}_{k-1}\right)\left(\begin{array}{c}
\alpha_{k-1} \\
1
\end{array}\right) \alpha_{k-1, h_{k}}^{-1}=\left(-R_{k-2} \alpha_{k-1}+\underline{r}_{k-1}\right) \alpha_{k-1, h_{k}}^{-1}=\underline{0},
\end{aligned}
$$

thus $R_{k-1} \varrho_{k}=-R_{k-2, h_{k}}$. Then

$$
\begin{aligned}
\underline{r}_{k} & =\left(-I_{m}, \underline{r}\right) B_{k}\left(\frac{0}{1}\right)=\left(-I_{m}, \underline{r}\right) B_{k-1} E_{h_{k}} A\left(\underline{a}_{k}\right)\left(\begin{array}{l}
\underline{0} \\
1
\end{array}\right) \\
& =\left(-R_{k-1},-R_{k-2, h_{k}}\right)\left(\begin{array}{c}
\underline{a}_{k} \\
1
\end{array}\right)=\left(-R_{k-1},-R_{k-2, h_{k}}\right)\left(\begin{array}{c}
\varrho_{k}-\alpha_{k} \\
1
\end{array}\right) \\
& =\left(-R_{k-1},-R_{k-2, h_{k}}\right)\left(\begin{array}{c}
-\alpha_{k} \\
0
\end{array}\right)=R_{k-1} \alpha_{k} .
\end{aligned}
$$

To prove $\operatorname{Iv}\left(R_{k-1} \alpha_{k}\right)=\operatorname{Iv}\left(D_{k} \alpha_{k}\right)$, we need to know the relation between $R_{k-1}$ and $D_{k}$. For this purpose we introduce two concepts: base matrix and $D$-component of a base matrix.

Definition 17. We call a square matrix $R$ of order $m$ over $F\left(\left(z^{-1}\right)\right)$ a base matrix if $R(j) \neq \underline{0}$ and $I(R(j))=j$ for each $1 \leq j \leq m$, where $R(j)$ denotes the $j$ th column of $R$. For a base matrix $R$, the $D$-matrix $\Delta=\operatorname{diag}\left(z^{-v_{1}}, \ldots, z^{-v_{m}}\right)$ is called the $D$-component of $R$ if $v_{j}=v(R(j))$ for each $j$.

Lemma 18. Let $R$ be a base matrix, and $\Delta$ the $D$-component of $R$. Then $R$ is invertible, and $\operatorname{Iv}(R \underline{r})=\operatorname{Iv}(\Delta \underline{r})$ for all $\underline{r} \in F\left(\left(z^{-1}\right)\right)^{m}$. 
Proof. Let $L=R \Delta^{-1}$. It is clear that $\operatorname{Iv}\left(L_{j}\right)=(j, 0)$ for all $1 \leq j \leq m$, where $L_{j}$ denotes the $j$ th column of $L$. It is enough to prove that $L$ is invertible, and $\operatorname{Iv}(L \underline{r})=\operatorname{Iv}(\underline{r})$ for all $\underline{r} \in F\left(\left(z^{-1}\right)\right)^{m}$, since then $R=L \Delta$ is invertible, and $\operatorname{Iv}(R \underline{r})=\operatorname{Iv}(L \Delta \underline{r})=\operatorname{Iv}(\Delta \underline{r})$.

It is clear that $v(\operatorname{det}(L))=0$, so $\operatorname{det}(L) \neq 0$, hence $L$ is invertible. Let $\operatorname{Iv}(\underline{r})=(h, v), L \underline{r}=\left(r_{1}^{\prime}, \ldots, r_{m}^{\prime}\right)^{\tau}, \underline{r}=\left(r_{1}, \ldots, r_{m}\right)^{\tau}, L=\left(s_{i, j}\right)$. Then $r_{i}^{\prime}=\sum_{j} s_{i, j} r_{j}$. Note that $v\left(s_{i, j}\right)>0$ for $j>i, v\left(s_{i, i}\right)=0$, and $v\left(s_{i, j}\right) \geq 0$ for $j<i$; $v\left(r_{j}\right)>v$ for $j<h, v\left(r_{h}\right)=v$, and $v\left(r_{j}\right) \geq v$ for $j>h$. It is easy to check that $v\left(r_{i}^{\prime}\right)>v$ for $i<h, v\left(r_{h}^{\prime}\right)=v, v\left(r_{i}^{\prime}\right) \geq v$ for $i>h$, based on Theorem 3. Hence, $\operatorname{Iv}(L \underline{r})=(h, v)=\operatorname{Iv}(\underline{r})$.

Lemma 19. $\operatorname{Iv}\left(R_{k-1, j}\right)=\left(j, v_{k, j}\right)$ for $0 \leq k \leq \mu$. In particular, $R_{k-1}$ is a base matrix, and $D_{k}$ is the $D$-component of $R_{k-1}$ for $0 \leq k \leq \mu$.

Proof. We reason by induction on $k$. When $k=0$, we have $R_{-1}=I_{m}$, so $R_{-1, j}=\underline{e}_{j}$, hence $\operatorname{Iv}\left(R_{-1, j}\right)=\operatorname{Iv}\left(\underline{e}_{j}\right)=(j, 0)=\left(j, v_{0, j}\right)$. Now assume $\operatorname{Iv}\left(R_{i-1, j}\right)=\left(j, v_{i, j}\right)$ for $0 \leq i<k$ and $1 \leq j \leq m$. In particular, we assume $\operatorname{Iv}\left(R_{k-2, j}\right)=\left(j, v_{k-1, j}\right)$, hence $R_{k-2}$ is a base matrix, and $D_{k-1}\left(=\Delta_{k-2}\right)$ is the $D$-component of $R_{k-2}$. If $j \neq h_{k}$, we have seen that $R_{k-1, j}=R_{k-2, j}$, so $\operatorname{Iv}\left(R_{k-1, j}\right)=\operatorname{Iv}\left(R_{k-2, j}\right)=\left(j, v_{k-1, j}\right)=\left(j, v_{k, j}\right)$. Since $R_{k-1, h_{k}}=-\underline{r}_{k-1}=$ $-R_{k-2} \alpha_{k-1}$, we conclude that

$$
\begin{aligned}
\operatorname{Iv}\left(R_{k-1, h_{k}}\right) & =\operatorname{Iv}\left(R_{k-2} \alpha_{k-1}\right)=\operatorname{Iv}\left(\Delta_{k-2} \alpha_{k-1}\right) \\
& =\left(h_{k}, c_{k}\right)=\left(h_{k}, c_{k, h_{k}}\right)=\left(h_{k}, v_{k, h_{k}}\right) .
\end{aligned}
$$

Proof of Proposition 15(2), (3). From Lemmas 18 and 19 we see immediately that $\operatorname{Iv}\left(R_{k-1} \alpha_{k}\right)=\operatorname{Iv}\left(D_{k} \alpha_{k}\right)$, which leads to item (2). From (1) and (2) we get

$$
\begin{aligned}
\operatorname{Iv}\left(\underline{r}_{k}\right) & =\operatorname{Iv}\left(R_{k-1} \alpha_{k}\right)=\operatorname{Iv}\left(D_{k} \alpha_{k}\right)=\operatorname{Iv}\left(D_{k} \alpha_{k}\right) \\
& =\operatorname{Iv}\left(\Delta_{k-1} \alpha_{k}\right)=\left(h_{k+1}, c_{k+1}\right)=\left(h_{k+1}, v_{k+1}\right),
\end{aligned}
$$

which is (3).

7.3. Proof of Theorem 12. The proof of Theorem 12 is based on the following lemma. Then

Lemma 20. Assume $0 \neq b_{i}(z) \in F[z], \operatorname{deg}\left(b_{i}(z)\right)<t_{i+1}, 0 \leq i \leq \mu$.

(1) $\operatorname{Iv}\left(\left\{\underline{r} q_{i}(z) b_{i}(z)\right\}\right)=\left(h_{i+1}, v_{i+1}-\operatorname{deg}\left(b_{i}(z)\right)\right)$.

(2) $\operatorname{Iv}\left(\left\{\underline{r} q_{i}(z) b_{i}(z)\right\}\right) \neq \operatorname{Iv}\left(\left\{\underline{r} q_{j}(z) b_{j}(z)\right\}\right), \forall 0 \leq j \neq i \leq \mu$ and $b_{i}(z) b_{j}(z)$ $\neq 0$.

Proof. (1) Since

$$
\operatorname{Iv}\left(\underline{r}_{i} b_{i}(z)\right)=\left(h_{i+1}, v_{i+1}-\operatorname{deg}\left(b_{i}(z)\right)\right)>\left(h_{i+1}, v_{i+1}-t_{i+1}\right) \geq\left(h_{i+1}, 0\right),
$$

we obtain $\left\{\underline{r}_{i} b_{i}(z)\right\}=\underline{r}_{i} b_{i}(z)$. Then 


$$
\left\{\underline{r} q_{i}(z) b_{i}(z)\right\}=\left\{\left(\underline{r} q_{i}(z)-\underline{p}_{i}\right) b_{i}(z)\right\}=\left\{\underline{r}_{i} b_{i}(z)\right\}=\underline{r}_{i} b_{i}(z) .
$$

So, $\operatorname{Iv}\left(\left\{\underline{r} q_{i}(z) b_{i}(z)\right\}\right)=\operatorname{Iv}\left(\underline{r}_{i} b_{i}(z)\right)=\left(h_{i+1}, v_{i+1}-\operatorname{deg}\left(b_{i}(z)\right)\right)$.

(2) If $h_{i+1} \neq h_{j+1}$, then (2) is an easy consequence of (1). If $h_{i+1}=h_{j+1}$, we may assume $j<i$. From (1) we have

$$
\begin{aligned}
v\left(\left\{\underline{r} q_{i}(z) b_{i}(z)\right\}\right) & =v_{i+1}-\operatorname{deg}\left(b_{i}(z)\right)>v_{i+1}-t_{i+1}=v_{i, h_{i+1}} \geq v_{j+1, h_{i+1}} \\
& =v_{j+1, h_{j+1}}=v_{j+1} \geq v_{j+1}-\operatorname{deg}\left(b_{j}(z)\right)=v\left(\left\{\underline{r} q_{j}(z) b_{j}(z)\right\}\right),
\end{aligned}
$$

which concludes the proof.

We can now prove Theorem 12. Set $d=\operatorname{deg}(q(z))$ and $(h, v)=\operatorname{Iv}(\{\underline{r} q(z)\})$. Since

$$
\operatorname{Iv}(\underline{r} q(z)-\underline{p}(z)) \leq \operatorname{Iv}(\{\underline{r} q(z)\}) \quad \text { and } \quad \underline{r}-\frac{\underline{p}(z)}{q(z)}=\frac{\underline{r} q(z)-\underline{p}(z)}{q(z)}
$$

we get $\operatorname{Iv}(\underline{r}-\underline{p}(z) / q(z)) \leq(h, v+d)$. It is enough to prove

$$
(h, v) \leq\left(h_{k+1}, n_{k+1}-d\right),
$$

since then we have $\operatorname{Iv}(\underline{r}-\underline{p}(z) / q(z)) \leq(h, v+d) \leq\left(h_{k+1}, n_{k+1}\right)$. With the assumption $d_{k} \leq d<\bar{d}_{k+1}$ we can write $q(z)=\sum_{0 \leq i \leq k} b_{i}(z) q_{i}(z)$ for some $b_{i}(z) \in F[z]$ such that $\operatorname{deg}\left(b_{i}(z)\right)<\operatorname{deg}\left(q_{i+1}(z)\right)-\operatorname{deg}\left(q_{i}(z)\right)=t_{i+1}$ for each $i \geq 0$ and $b_{i}(z) \neq 0$ (note that $q_{0}(z)=1$ ) and $\operatorname{deg}\left(b_{k}(z)\right)=d-d_{k} \geq 0$. It is clear that $\{\underline{r} q(z)\}=\sum_{0 \leq i \leq k}\left\{\underline{r} q_{i}(z) b_{i}(z)\right\}$. From Lemmas 20 and 3 we have

$$
\begin{aligned}
(h, v) & =\operatorname{Iv}(\{\underline{r} q(z)\})=\min \left\{\operatorname{Iv}\left(\left\{\underline{r} q_{i}(z) b_{i}(z)\right\}\right) \mid b_{i}(z) \neq 0,0 \leq i \leq k\right\} \\
& \leq \operatorname{Iv}\left(\left\{\underline{r} q_{k}(z) b_{k}(z)\right\}\right)=\left(h_{k+1}, v_{k+1}-\operatorname{deg}\left(b_{k}(z)\right)\right) \\
& =\left(h_{k+1}, v_{k+1}+d_{k}-d\right)=\left(h_{k+1}, n_{k+1}-d\right) .
\end{aligned}
$$

8. Remark. We have focused on m-CFA in this paper. We showed that the m-CFA produces a multi-continued fraction expansion $C(\underline{r})$ of $\underline{r}$ for any multiple Laurent series $\underline{r}$, which provides optimal rational approximations to $\underline{r}$.

For further study, consider an arbitrary data of the expansion form

$$
C=\left[\underline{0}, h_{1}, \underline{a}_{1}, \ldots, h_{k}, \underline{a}_{k}, \ldots\right], \quad 1 \leq h_{k} \leq m, \underline{a}_{k} \in F[z]^{m}, 1 \leq k \leq \mu,
$$

which satisfies the three conditions formulated for $C(\underline{r})$ in Section 5 . We call such a $C$ a multi-continued fraction. From the definition we see that multi-continued fractions are not necessarily identical to $C(\underline{r})$ for some $\underline{r}$. The problem arises whether multi-continued fractions have similar properties to those of $C(\underline{r})$, to be specific, whether any multi-continued fraction $C$ converges to an element $\underline{r}$ in $F\left(\left(z^{-1}\right)\right)^{m}$ and provides optimal rational approximations to $\underline{r}$, and whether one can construct an algorithm which produces such $C$. The answers to these problems are affirmative, and we will call the expected algorithm the multi-universal continued fraction algorithm (m-UCFA, for short). We will discuss these problems in another paper. 
Acknowledgments. The authors wish to thank the referees for pointing out the references $[5,9,11,12]$, and for the detailed comments and suggestions that improved this paper.

\section{References}

[1] L. Bernstein, The Jacobi-Perron Algorithm-Its Theory and Application, Lecture Notes in Math. 207, Springer, Berlin, 1971.

[2] K. Feng and F. Wang, The Jacobi-Perron algorithm on function fields, Algebra Colloq. 1 (1994), 149-158.

[3] T. W. Hungerford, Algebra, Springer, 1974.

[4] K. Inoue, On the exponential convergence of Jacobi-Perron algorithm over $\mathbb{F}(x)^{d}$, JP J. Algebra Number Theory Appl. (3) 1 (2003), 27-41.

[5] K. Inoue and H. Nakada, The modified Jacobi-Perron algorithm over $\mathbb{F}_{q}(X)^{d}$, Tokyo J. Math. 26 (2003), 447-470.

[6] S. Ito, J. Fujii, H. Higashino and S.-I. Yasutomi, On simultaneous approximation to $\left(\alpha, \alpha^{2}\right)$ with $\alpha^{3}+k \alpha-1=0$, J. Number Theory 99 (2003), 255-283.

[7] S. Ito, M. Keane and M. Ohtsuki, Almost everywhere exponential convergence of the modified Jacobi-Perron algorithm, Ergodic Theory Dynam. Systems 13 (1993), 319-334.

[8] A. Lasjaunias, Diophantine approximation and continued fraction expansions of algebraic power series in positive characteristic, J. Number Theory 65 (1997), 206-225.

[9] -, A survey of Diophantine approximation in fields of power series, Monatsh. Math. 130 (2000), 211-229.

[10] R. Meester, A simple proof of the exponential convergence of the modified JacobiPerron algorithm, Ergodic Theory Dynam. Systems 19 (1999), 1077-1083.

[11] R. Paysant-Le Roux et E. Dubois, Algorithme de Jacobi-Perron dans un corps de séries formelles, C. R. Acad. Sci. Paris Sér. A 272 (1971), 564-566.

[12] - - Étude métrique de l'algorithme de Jacobi-Perron dans un corps de séries formelles, ibid. 275 (1972), 683-686.

[13] E. V. Podsypanin, A generalization of continued fraction algorithm that is related to Viggo Brun algorithm, Zap. Nauchn. Sem. Leningrad. Otdel. Mat. Inst. Steklov. 67 (1977), 184-194 (in Russian).

[14] W. M. Schmidt, On continued fractions and diophantine approximation in power series fields, Acta Arith. 95 (2000), 139-166.

[15] H. Stichtenoth, Algebraic Function Fields and Codes, Springer, 1991.

State Key Laboratory of Information Security

Graduate School of Chinese Academy of Science

Beijing, 100039, P.R. China

E-mail: yangdai@public.bta.net.cn

kunpengwang@263.net

ydf@is.ac.cn

Received on 9.8.2004

and in revised form on 17.11.2005 